\title{
I nfecção por Chlamydia trachomatis no período neonatal: aspectos clínicos e laboratoriais. Experiência de uma década: 1987-1998
}

\author{
F.A.C. VAZ, M.E.J. CECCON, E.M.A. DINIZ
}

Unidade de Cuidados I ntensivos Neonatal- Instituto da Criança do Hospital das Clínicas da FMUSP - Departamento de Pediatria da FMUSP, São Paulo, SP.

RESUMO - A infecção por C. trachomatis é adqui rida pelo recém-nascido ( $\mathrm{R} N$ ) principalmente durante sua passagem pelo canal do parto; $25 \%$ a $50 \%$ destes deverão desenvolver conjuntivite e $10 \%$ a $20 \%$ pneumonia.

Овјетıvos. Verificar a incidência de infecção ocular por C. trachomatis nos R N internados com diagnóstico de conjuntivite, num período de $\mathbf{1 0}$ anos. - Observar a associação entre infecção ocular é pneumonia intersticial - Estudar os aspectos epidemiológicos e os métodos utilizados para o diagnóstico laboratorial.

Casuística e Metodologia. Foram analisados os R N internados com diagnóstico de conjuntivite e/ ou pneumonia intersticial internados na UCINE no período de 1987-1998. Os métodos de diagnóstico utilizados foram: a pesquisa direta do agente etiológico em raspado de conjuntiva, radiografia de tórax, sorologia para C. trachomatis no sangue pelo método de imunofluorescência para anticorpos IgG e IgM.

Resultados. Estudamos as características de $\mathbf{2 0}$ RN que apresentaram infecção por $C$. trachomatis : 15 eram de termo ( $75 \%$ ) e cinco, pré-termos

\section{INTRODUÇÃO}

O gênero chlamydia compreende um grupo de parasitas intracelulares obrigatórios, três espécies sendo reconhecidas: C. psitacci, C. pneumoniae (cepa TWAR) e C. trachomatis; a primeira delas é um patógeno comum em espécies de aves e mamíferos e as duas últimas, causadoras de doenças em seres humanos ${ }^{1,2}$.

As clamídias são classificadas como bactérias gram-negativas, altamente especializadas. O sorotipo Twar da C. pneumoniae causa bronquite, pneumonia e doença arterial. Em relação a C. trachomatis, 15 sorotipos são reconhecidos: L 1, L2 e $L 3$, causadores do linfogranuloma venéreo; $A, B$, B a e C, causadores do tracoma ocular; D,E,F, G, e $H$, causadores da conjuntivite de inclusão observada no recém-nascido (RN) e os sorotipos I,J e $K$, causadores da pneumonia do recém-nascido $(R N)$ e
(25\%); houve predominância da infecção no sexo feminino (60\%); a pneumonia esteve presente em 15 dos 20 R N (75\%) e 12 apresentaram associação de conjuntivite e pneumonia. Não houve relação significante entre tipo de parto, idade materna, número de parceiros e a infecção, sendo que o antecedente materno de leucorreia esteve presente em $\mathbf{5 0} \%$ dos casos. 0 diagnóstico sorológico esteve relacionado com a presença de pneumonia e a pesquisa direta com a conjuntivite. A incidência de conjuntivite por $C$. trachomatis entre os RN internados com esse diagnóstico durante o período de estudo foi de 17/100 (17\%).

Conclusõos. A C. trachomatis é um importante agente patogênico e sua pesquisa é muito importante em RN com conjuntivite e/ou pneumonia intersticial mesmo na ausência de fatores de risco para doença sexualmente transmissível. A pesquisa direta em raspado de conjuntiva e o exame sorológico se mostraram importantes como métodos auxiliares do diagnóstico.

UNITERMOS: Chlamydia trachomatis. Conjuntivite neonatal. Pneumonia intersticial. do lactente, da uretrite, da cervicite, da sal pingite e da epididimite ${ }^{3,4}$.

A epidemiologia da infecção perinatal por C. trachomatis foi descrita décadas antes do isolamento do microrganismo. Halberstaedter e Prowasek visualizaram pela primeira vez as inclusões intracitoplasmáticas em 1907 e Linder et al. nos anos de 1909-1911 descreveram o mesmo padrão citol ógi co em infecções oculares não gonocócicas e em secreções do trato genital de mães que geraram crianças infectadas ${ }^{3}$.

A C. trachomatis é provavel mente a infecção sexualmente transmissível mais prevalente nos Estados Unidos ${ }^{5}$ e o seu Centro de Controle de Doenças (CDC) estima que o número de novas infecções por este microrganismo exceda quatro milhões anualmente $e^{6,7}$.

A gestação por si só parece aumentar o risco de col onização por essa bactéria ${ }^{8}$. Os fatores de risco, 


\begin{tabular}{|c|c|c|c|c|c|c|c|c|c|}
\hline \multirow{2}{*}{$\begin{array}{c}\text { Caso } \\
\text { № }\end{array}$} & \multirow[t]{2}{*}{ Nome } & \multicolumn{2}{|c|}{ TIPO DE PARTO } & \multirow{2}{*}{$\begin{array}{l}\text { Idade } \\
\text { materna } \\
\text { (anos) }\end{array}$} & \multicolumn{2}{|c|}{ PRÉ-NATAL } & \multicolumn{2}{|c|}{ RPM > 12 HORAS } & \multirow{2}{*}{$\begin{array}{c}\text { № } \\
\text { parceiros }\end{array}$} \\
\hline & & NORMAL & CESÁREA & & SIM & NÃO & SIM & NÃO & \\
\hline 1 & DBS & & + & 25 & + & & + & & 1 \\
\hline 2 & PBO & + & & 20 & & + & + & & 1 \\
\hline 3 & TRC & & + & 19 & & + & & + & 1 \\
\hline 4 & MEA & & + & 28 & & + & + & & 1 \\
\hline 5 & MCV & + & & 23 & & + & + & & 1 \\
\hline 6 & RDS & + & & 18 & + & & + & & $+\mathrm{DE} 1$ \\
\hline 7 & HDA & & + & 20 & + & & + & & 1 \\
\hline 8 & NDS & & + & 23 & & + & & + & $+\mathrm{DE} 1$ \\
\hline 9 & MAS & + & & 25 & & + & & + & $+\mathrm{DE} 1$ \\
\hline 10 & MCB & & + & 25 & & + & & + & 1 \\
\hline 11 & MPS & + & & 18 & & + & & + & 1 \\
\hline 12 & NAO & + & & 27 & & + & & + & 1 \\
\hline 13 & CAS & + & & 17 & + & & + & & 1 \\
\hline 14 & GSN & + & & 23 & + & & + & & 1 \\
\hline 15 & DS & & + & 20 & + & & & + & 1 \\
\hline 16 & FSN & & + & 21 & & + & & + & 1 \\
\hline 17 & FCF & + & & 22 & & + & + & & $+\mathrm{DE} 1$ \\
\hline 18 & IAP & + & & 30 & + & & + & & 1 \\
\hline 19 & SS & + & & 18 & + & & & + & 1 \\
\hline 20 & $\mathrm{CHS}$ & & + & 37 & + & & & + & 1 \\
\hline $\begin{array}{l}\text { Parto r } \\
\text { Ruptur } \\
\text { Mais d }\end{array}$ & $\begin{array}{l}\text { al: } 11 / 20 \\
\text { ematura } \\
\text { parceir }\end{array}$ & $\begin{array}{l}, 0 \%) \text { Sem pr } \\
\text { nembranas > } \\
20(20 \%) \mathrm{M}\end{array}$ & $\begin{array}{l}\text { ré-natal } 11 / 20 \\
12 \text { horas } 9 / 2 \\
\text { ãe com idade }\end{array}$ & $\begin{array}{l}\% \%) \\
45 \%) \\
20 \text { anos 5/ }\end{array}$ & & & & & \\
\hline
\end{tabular}

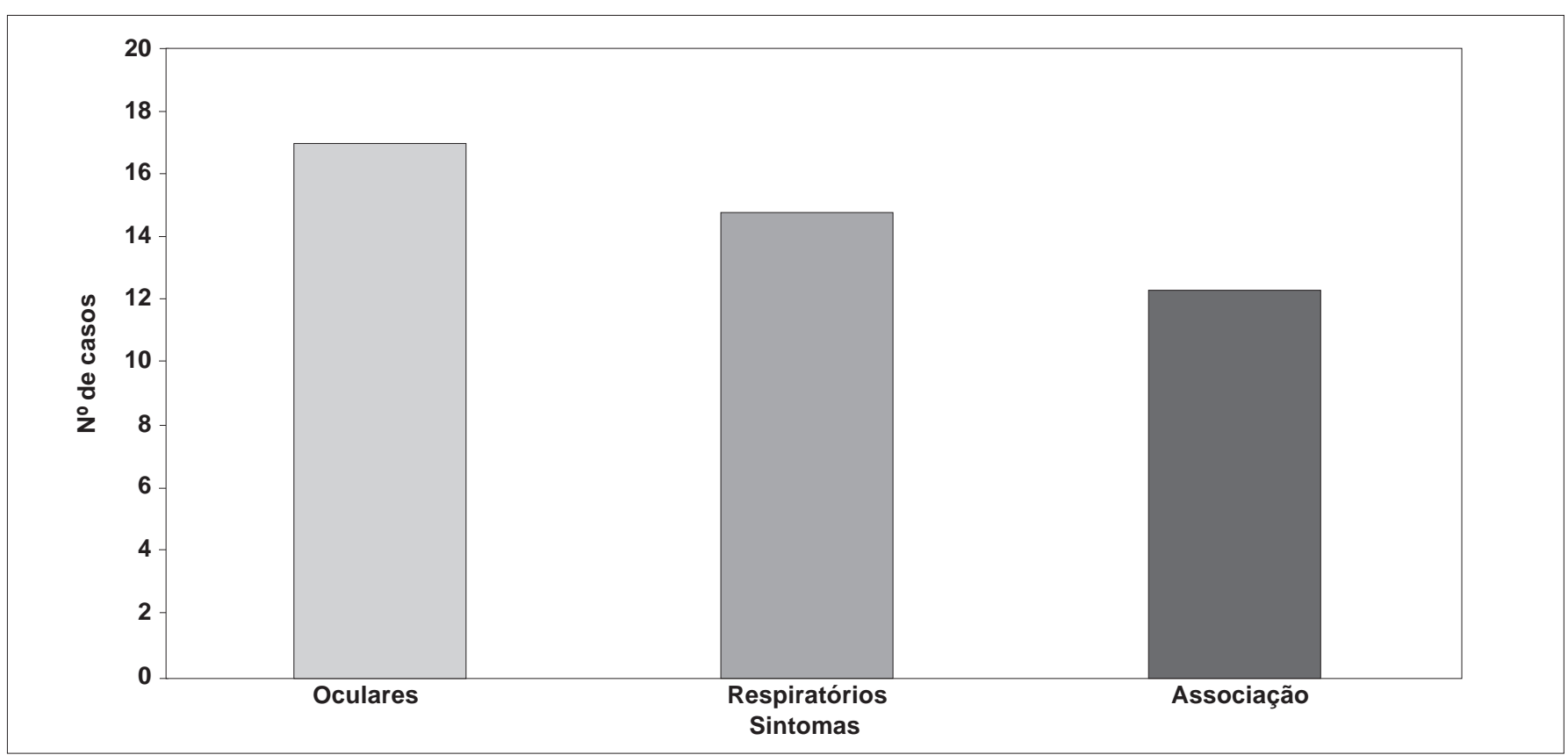

Gráfico 1 - Características clínicas de 20 RN com infecção por C. trachomatis

além da própria gestação, são a vida sexual ativa, baixas condições socio-econômicas, vários parceiros e antecedentes de outras doenças sexualmente transmissíveis ${ }^{9-11}$.

A infecção por $C$. trachomatis é adquirida pelo RN durante a passagem pelo canal do parto, existindo casos de infecção em crianças nascidas de parto cesárea ${ }^{12}$ com antecedentes maternos de ruptura prematura de membranas amnióticas, e, mesmo a pós-natal sendo possível através do contato com a mãe ${ }^{13}$. O RN de mãe com infecção por C. tracomatis na cervix uterina tem $60 \%$ a $70 \%$ de 


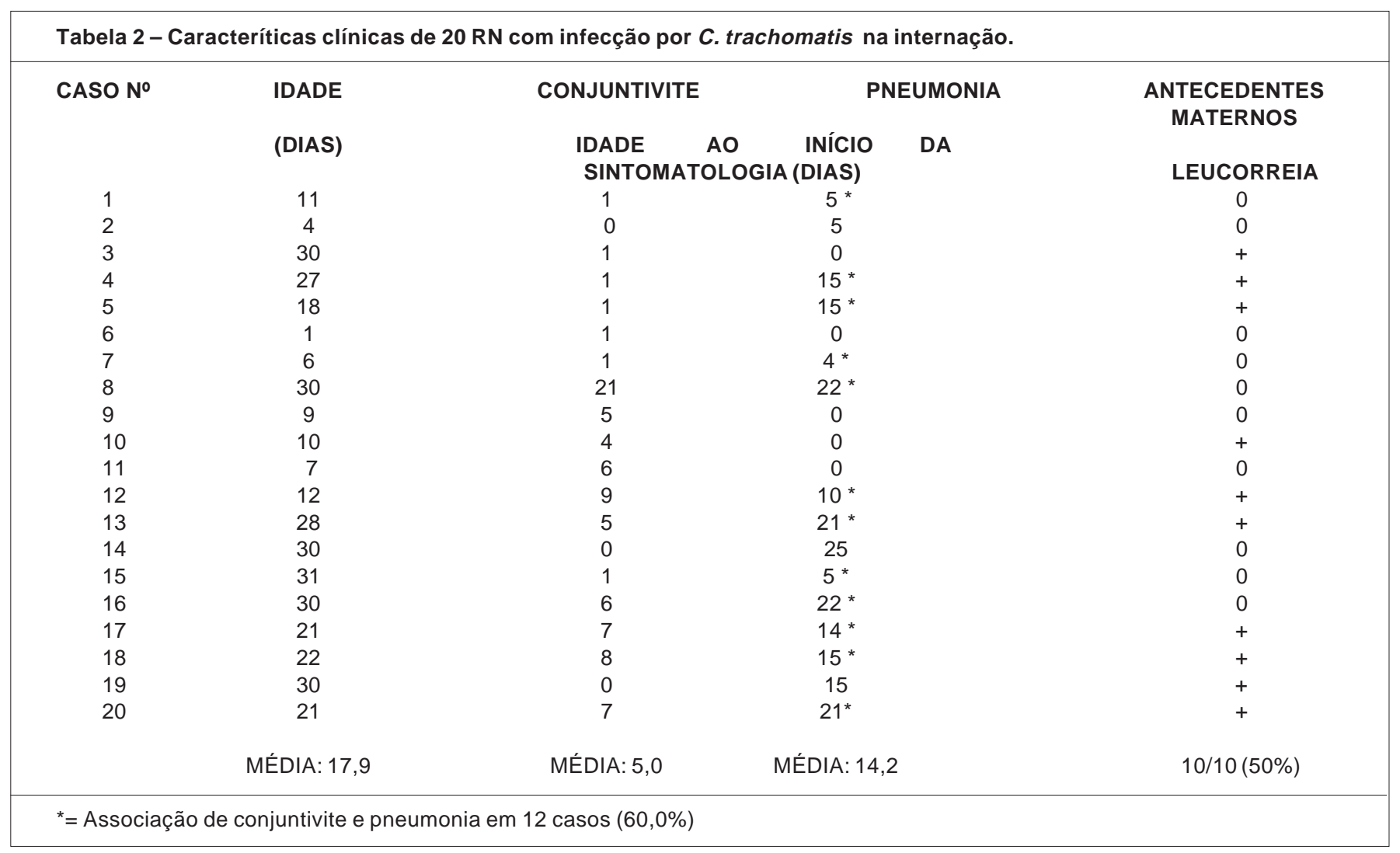

risco de adquirir a infecção durante sua passagem pelo canal do parto: $25 \%$ a $50 \%$ deverão desenvolver conjuntivite e $10 \%$ a $20 \%$ pneumonia ${ }^{14,15}$. A infecção ocular manifesta-se entre cinco e 12 dias após o nascimento e o quadro pulmonar, na maior parte dos casos, antes da oitava semana de vida ${ }^{16}$.

Em nosso meio, o diagnóstico pré-natal de mães portadoras de infecção por esta bactéria é difícil, uma vez que a pesquisa não faz parte dos exames de rotina. Nos últimos anos, com a melhora dos métodos de diagnóstico através de exames diretos e sorológicos para C. trachomatis, foi possível reconhecer no RN e no lactente tanto a conjuntivite de inclusão como a pneumonia. Esses exames são realizados em todos os RN com suspeita dessas patologias na Unidade de Cuidados I ntensivos Neonatal (UCINE) do Instituto da Criança do Hospital das Clínicas da FMUSP.

O reconhecimento dessa infeç̧ão tem implicações diretas no tratamento do paciente e dos pais, bem como seguimento a longo prazo das crianças contaminadas, pois, segundo Bell et al. (1992), a doença pulmonar pode evoluir para doença pulmonar obstrutiva crônica5,18.

\section{OBJ ETIVOS}

Os objetivos deste trabal ho foram: verificar a incidência de infecção ocular por Chlamydia tra- chomatis nos RN internados com diagnóstico de conjuntivite, num período de 10 anos (1997-1998); verificar a associ ação entre infecção ocular e pneumonia intesticial nos RN infectados e estudar os aspectos epidemiológicos e os métodos utilizados para o diagnóstico laboratorial.

\section{CASUÍSTICA E METOdOLOGIA}

Foram estudados retrospectivamente $20 \mathrm{RN}$ que apresentaram diagnóstico de infecção neonatal por C. trachomatis, dentre aqueles internados na UCINE com diagnóstico de conjuntivite e/ou pneumonia no período proposto para o estudo. F oram analisados os dados epi demi ol ógi cos importantes para a ocorrência dessa infecção a partir da história clínica específica para doenças sexualmente transmissíveis.

Fizeram parte dos métodos de diagnóstico: a pesquisa dir reta do agente etiológico em raspado de conjuntiva, radiografia de tórax, sorologia para Chlamydia trachomatis no sangue pelo método de imunofluorescencia para anticorpos IgG e IgM.

\section{RESULTADOS}

Dentre os $20 \mathrm{RN}$ estudados, 15 eram de termo ( $75 \%$ ) e cinco pré-termos ( $25 \%)$, sendo 12 do sexo 
Tabela 3 - Exames auxiliares e terapêutica administrada em 20 RN cominfecção por C. trachomatis.

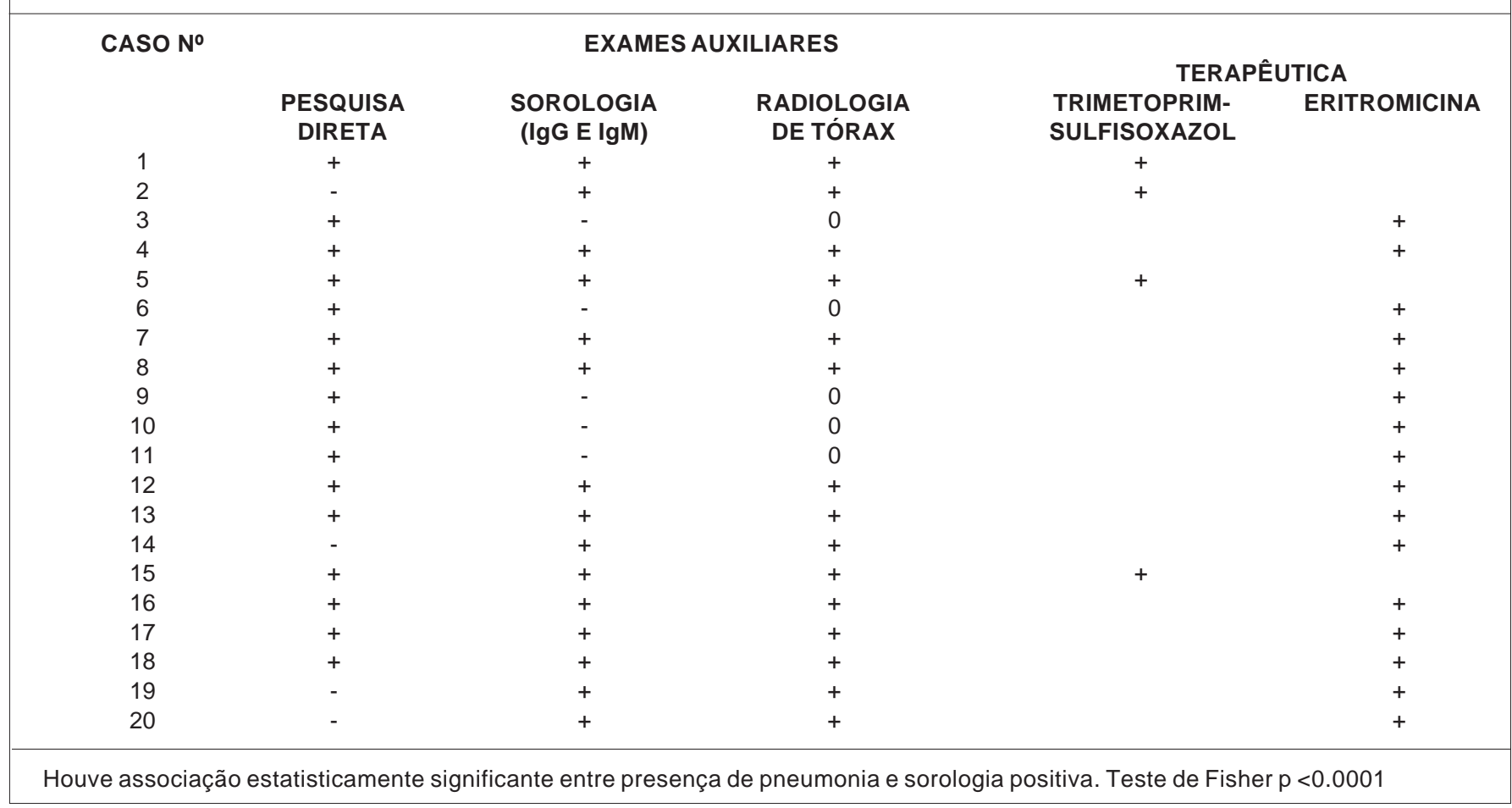

feminino (60\%) e oito (40) do sexo masculino.

A incidência de conjuntivite por $C$. trachomatis entre os RN internados com essa patologia no período estudado foi de 17/100 (17\%). Apresentamos na forma de tabelas $(1,2,3,4,5)$ e gráfico (1), outros dados dos RN estudados.

\section{DISCUSSÃO}

\section{Aspectos clínicos: conjuntivite}

No RN os principais agentes etiológicos que causam inflamação da conjuntiva são as bactérias (Neisseria gonorrhoeae, Chlamydia trachomatis, Streptococcus sp, Staphylococcus aures ou epidermidis, E. coli e outras bactérias gram-negativas), as drogas oculares (conjuntivite química) e os vírus do tipo Herpes. Com a profilaxia realizada com solucões oculares tópicas ao nascimento (nitrato de prata a $1 \%$, eritromicina $0,5 \%$ e tetraticlina $1 \%$ ) a incidência da patologia infecciosa diminuiu, ocorrendo ainda ao redor de $20 \%$ de todos os RN.

A conjuntivite freqüentemente encontrada atualmente é a provocada pela $C$. trachomatis e é conhecida como conjuntivite de inclussão em decorrência dos achados anátomo-patológi $\cos ^{19}$. E mbora essa bactéria seja susceptível aos medicamentos tópicos citados, a eficácia da profilaxia tópica não é bem estabel ecida. Hamerslag et al. ${ }^{10}$ mostraram ser a eritromicina mais eficaz do que o nitrato de prata para essa bactéria e menos eficaz para o gonococo. Por ser esta última bactéria de maior prevalência e mais grave, a profilaxia da conjuntivite continua a ser efetuada, na maioria dos hospitais, pela sol ução de nitrato de prata a 1\%.

A C. trachomatis é adquirida pelo RN na maior parte das vezes durante a passagem pelo canal de parto. Em nossa casuística, 55\% dos RN haviam nascido de parto normal e $45 \%$ de parto cesárea (Tabela 1), fato sugesti vo de que o ti po de parto não invalida o diagnóstico, conforme também é descrito na literatura ${ }^{20}$.

A incidência de conjuntivite por $\mathrm{C}$. trachomatis entre os RN internados com esse diagnóstico no período de estudo foi de 17\% (17/100). Sabe-se pela literatura que $25 \%$ a $50 \%$ dos fil hos de mães portadoras de C. trachomatis terão conjuntivite. N esta casuística, não temos esse dado materno, porém dos 17 RN com este diagnóstico, em nove (52,3\%) as mães tinham antecedentes de leucorréia (TabeIa 2), achado que indiretamente sugere a presença de infecção por essa bactéria ${ }^{14}$. A média de início da sintomatologia nos $17 \mathrm{RN}$ foi com cinco dias de vida, (tabela 2) o que também está de acordo com a literatura, pois, o ciclo do microrganismo sendo de 48 horas, o tempo de início da sintomatologia é mais tardia em relação à de outros microrganismos, ou seja, entre cinco e 14 dias. Por outro lado, o achado de conjunti vite com início no primeiro dia 


\begin{tabular}{|c|c|c|c|c|c|c|c|c|c|c|}
\hline $\begin{array}{l}\text { CASO } \\
\text { № }\end{array}$ & $\begin{array}{c}\mathrm{Ht} \\
(\mathrm{g} \%)\end{array}$ & $\begin{array}{l}\text { HT } \\
(\%)\end{array}$ & $\begin{array}{l}\text { LEUC. } \\
\left(\mathrm{mm}^{3}\right)\end{array}$ & $\begin{array}{c}\text { BAST. } \\
(\%)\end{array}$ & $\begin{array}{c}\text { SEG. } \\
(\%)\end{array}$ & $\begin{array}{l}\text { IND. } \\
\text { NEUT. }\end{array}$ & $\begin{array}{c}\text { EOS. } \\
(\%)\end{array}$ & $\begin{array}{l}\text { LINF. } \\
(\%)\end{array}$ & $\begin{array}{c}\text { MONOC } \\
(\%)\end{array}$ & $\begin{array}{l}\text { PLAQ. } \\
\left(\mathrm{mm}^{3}\right)\end{array}$ \\
\hline 1 & 12,4 & 38,0 & 26.700 & 16,0 & 65,0 & 0,19 & 0,0 & 17,0 & 2 & 200.000 \\
\hline 2 & 13,2 & 46,0 & 12.900 & 17,0 & 44,0 & 0,27 & 2,0 & 46,0 & 3,0 & 153.000 \\
\hline 3 & - & - & - & - & - & - & - & - & - & - \\
\hline 4 & 11,7 & 36,0 & 8.600 & 2,0 & 64,0 & 0,03 & 2,0 & 31,0 & 0 & 210.000 \\
\hline 5 & 12,0 & 35,0 & 10.3000 & 11,0 & 66,0 & 0,14 & 0,0 & 22,0 & 0 & 190.000 \\
\hline 6 & 17,1 & 49,0 & - & - & - & - & - & - & - & - \\
\hline 7 & 14,6 & 43,2 & 7.000 & 6,0 & 21,0 & 0,22 & 0,0 & 71,2 & 2,0 & 200.000 \\
\hline 8 & 10,8 & 32,0 & 8.500 & 2,0 & 36,0 & 0,05 & 0,0 & 54,0 & 2,0 & 150.000 \\
\hline 9 & 10,8 & 32,0 & 10.400 & - & - & - & - & - & - & 556.000 \\
\hline 10 & - & - & - & - & - & - & - & - & - & - \\
\hline 11 & 17,0 & 51,5 & 17.302 & 1,0 & 63,0 & 0,01 & 0,0 & 35,0 & 1,0 & 156.000 \\
\hline 12 & 10,9 & 32,0 & 15.000 & 3 & 72,0 & 0,04 & 0,0 & 23,0 & 0,0 & 274.000 \\
\hline 13 & 11,6 & 33,0 & 18.000 & 6,0 & 30,0 & 0,16 & 6,0 & 53,0 & 5,0213 & 380.000 \\
\hline 14 & 10,0 & 30,3 & 17.300 & 1,0 & 15,0 & 0,06 & 2,0 & 79,0 & 3,0 & 213.000 \\
\hline 15 & - & - & - & - & - & - & - & - & - & - \\
\hline 16 & 11,5 & 33,0 & 17.500 & 2,0 & 58,0 & 0,03 & 2,0 & 35,0 & 1,0 & 340.000 \\
\hline \multicolumn{11}{|l|}{17} \\
\hline \multicolumn{11}{|l|}{18} \\
\hline \multicolumn{11}{|l|}{19} \\
\hline 20 & 12,4 & 34,0 & 14.800 & 1,0 & 42,0 & 0,02 & 2,0 & 46,0 & 3,0 & 314.000 \\
\hline
\end{tabular}

não afasta o diagnóstico. Essa situação ocorreu com os casos $1,3,4,5,6,7$ e 15 do nosso estudo, podendo o início precoce estar associado à ruptura prematura de membranas, citada em cinco desses RN (Tabela 1).

A média de idade em dias no início da internação foi de 17,9 (Tabela 2); verificamos, portanto, que o motivo desta, na mai oria dos casos, foi o quadro pulmonar e não somente a conjuntivite. É citado por Heggie et al. (1981) ${ }^{16}$ que essa doença acomete mais RN pré-termo, podendo isso ser atribuído, como os autores referem, ao maior tempo de internação desse tipo de criança com o conseqüente reconhecimento da doença ${ }^{21}$. No estudo presente a maioria dos $\mathrm{RN}$ foi de termo (75\%), tendo sido reinternados após alta da maternidade em UTI neonatal, o que permitiu reconhecer essa infecção. Acreditamos, assim, que não deve existir diferença em relação à idade gestacional. Na literatura não é citado predominância de sexo, porém, neste estudo a infecção predominou no sexo feminino (60\%.).

Esse tipo de conjuntivite de inclusão apresentase de forma aguda, com edema palpebral e secreção purulenta em moderada quantidade; inicialmente o comprometimento é unilateral, evolui em poucos dias para o olho contra-lateral, poucas vezes acometendo a córnea, porém, se não tratada, poderá evoluir com formação de "panos" e cicatrizes, prejudicando a visão a longo prazo. 0 atraso da medicação favorece indubitavel mente o aparecimento de seqüelas ${ }^{22}$.

Para evitar esse tipo de infecção seriam necessários exames periódicos durante o pré-natal para tratar a mãe e o seu parceiro. O antecedente de mães que realizaram pré-natal foi importante na composição da nossa casuística (45\%), (Tabela 1), porém, não contribuiu para evitar o aparecimento da infecção nos RN, já que não é feita a pesquisa de rotina durante as consultas de pré-natal.

\section{PNE UMONIA}

As infecções pulmonares persistem como causa importante de morbidade e mortal idade princi palmente nos países subdesenvolvidos ${ }^{23}$. $\mathrm{Na}$ Unidade de Cui dados I ntensivos Neonatal, reconhecendo a importância de verificar os agentes etiológicos da pneumonia do RN, existe uma rotina diagnóstica na qual pesquisam-se vírus, bactérias e fungos em crianças internadas com esse diagnóstico. São citados como fatores de risco para infecção por C. trachomatis aqueles comuns às doenças sexualmente transmissíveis, tais como a idade materna (mais freqüente em adol escentes), e múl ti plos parcei $\operatorname{ros}^{7,24}$. No estudo apresentado, podemos observar (Tabela 1) que a idade materna variou entre 17 e 37 anos e em $20 \%$ dos casos houve mais de um 

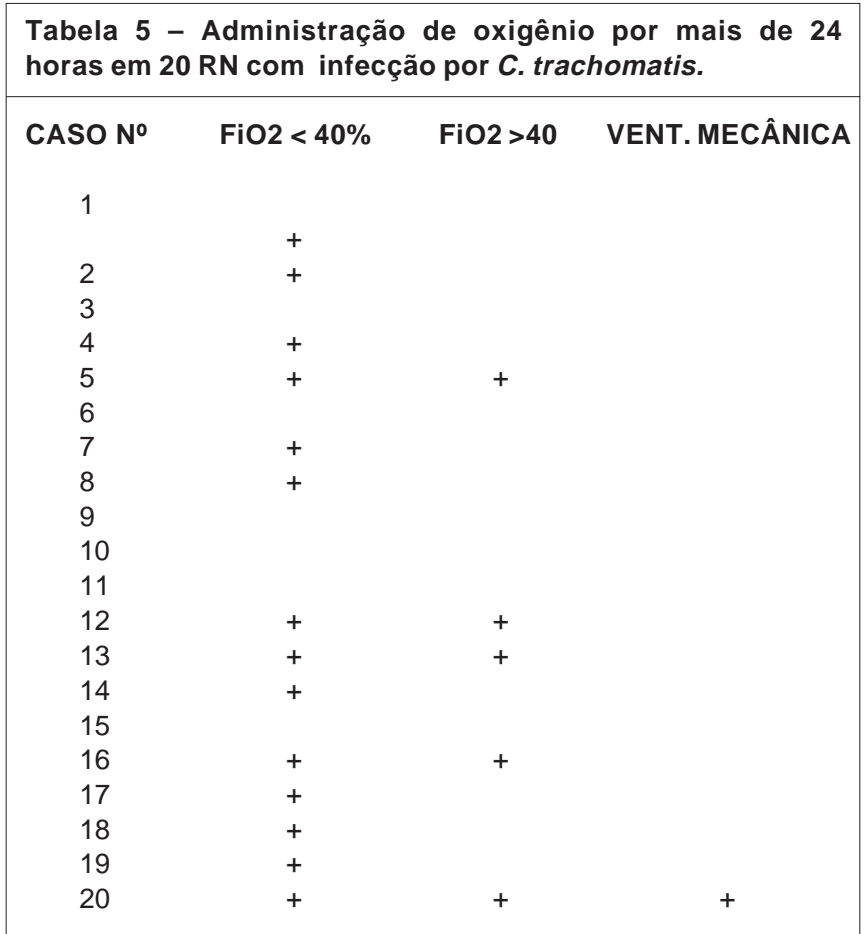

$\mathrm{FiO} 2<40 \%$ : 9/15 RN com quadro pulmonar

$\mathrm{FiO} 2<40 \%+\mathrm{FiO} 2>40 \% 4 / 15$

$\mathrm{FiO} 2<40 \%+\mathrm{FiO} 2>40 \%+$ VENT. MEC. $1 / 20$

CASOS: $3,6,9,10,11$ não tiveram pneumonia

CASO 20: sorologia + também para virus sincicial respiratório

parceiro. A falta de alguns fatores de risco comuns às doenças sexual mente transmissíveis não deve afastar o diagnóstico.

A ocorrência de pneumonia é de $10 \%$ a $20 \%$ nos RN de mães portadoras de $C$. trachomatis, sendo a nasofaringe posterior do R N olocal preferencial de sua replicação, e as culturas desse local costumam ser positivas enquanto que noutros locais (conjuntiva e faringe oral) podem ser negativas ${ }^{25}$. A história de conjuntivite não é um pré-requisito para o aparecimento de pneumonia; parece o que o trato respiratório pode ser diretamente infectado durante o nascimento ${ }^{26}$. Neste estudo, a pneumonia esteve presente em 15/20 RN (75\%), sendo que em 12 casos havia associação de conjuntivite e pneumonia e, em três casos, havia pneumonia sem antecedentes de conjuntivite.

Parece que a pneumonia no $\mathrm{R} N$ está relacionada a uma resposta de hipersensi bilidade, já que esses RN apresentam níveis el evados de anticorpos IgG para C. trachomatis. As mães infectadas também tem níveis el evados de anticorpos I gG, porém não parece ter efeito protetor, pois os RN, mesmo assim, adquirem a doença ${ }^{27}$.

A pneumonia por C. trachomatis foi descrita por Schacter et al. ${ }^{28}$ em 1975 em uma criança pósrecuperação de uma conjuntivite de inclusão. Os achados clínicos, descritos por Benn; Saxon ${ }^{26}$,em 1977, manifestam-se geralmente entre a segunda e 16ạ semana de vida no RN; o quadro respiratório costuma ser mais grave que no lactente, com aumento progressivo da freqüência respiratória (entre 50 e 70 movimentos respiratórios/minuto), cianose, deterioração do estado geral à ausculta pulmonar, roncos e estertores crepitantes difusos ${ }^{29,30}$. Na casuística apresentada, a média de início dos sintomas respiratórios foi de 14,2 dias, sendo que em quatro casos (1,2,7 e 15) ( Tabela 2) o início foi na primeira semana e os RN tiveram quadro ocular iniciado no primeiro dia de vida, com exceção do caso 2 que só teve pneumonia.

Dos 15 RN que tiveram pneumonia, 14 necessitaram de oxigênio por mais de 24 horas (Tabela 5), dos quais 9/14 com oxigênio inferior a $40 \%$ (na incubadora), 4/14 com oxigênio superior a 40\% (capacete) e um del es (caso 20) necessitou de ventilação mecânica. N esse último R N foi verificada a presença de vírus sincicial fator, de piora da evolução. O curso desta pneumonia é prolongadoe os sintomas persistem por mais de uma semana. Os RN desta casuística tiveram uma boa evolução, com tratamento sistêmico e obtiveram alta após completar antibioticoterapia.

\section{ASPECTOS LABORATORIAIS}

Para a pesquisa de $C$. trachomatis em conjuntiva, o material deve ser obtido por raspado da conjuntiva, com a pálpebra inferior evertida e corado pela técnica de GIEMSA.

Neste material são observadas inclusões azuis ou púr puras no citoplasma das células endoteliais; a sensibilidade do método é boa, atingindo $90 \%{ }^{31}$. Na casuística apresentada, a pesquisa de células de inclusão em material de conjuntiva foi positiva em todos os casos que apresentaram conjuntivite (17/20) (Tabela 3).

O diagnóstico sorológico pode ser realizado através de detecção de anticorpos específicos para C. trachomatis pelo método de imunofluorescen$\mathrm{cia}^{32}$. Os anticorpos da classe I gG têm origem através da passagem transplacentária e sua positividade no RN não significa infecção. Por outro lado, o encontro de anticorpos da classe I gM é de produção do próprio RN e diagnostica a infecção aguda ${ }^{33}$. Outro fato importante reside em que as crianças com infecções pulmonares apresentam taxas de anticorpos séricos superiores àquelas com infecção ocular ${ }^{25}$. Neste estudo todos os RN com pneumonia tiveram níveis de I gM mai or ou igual a $1 / 32$ (infecção aguda) e, aquel es que só ti veram conjuntivite, apresentaram sorologia negativa; houve associação estatisticamente significante (teste de 


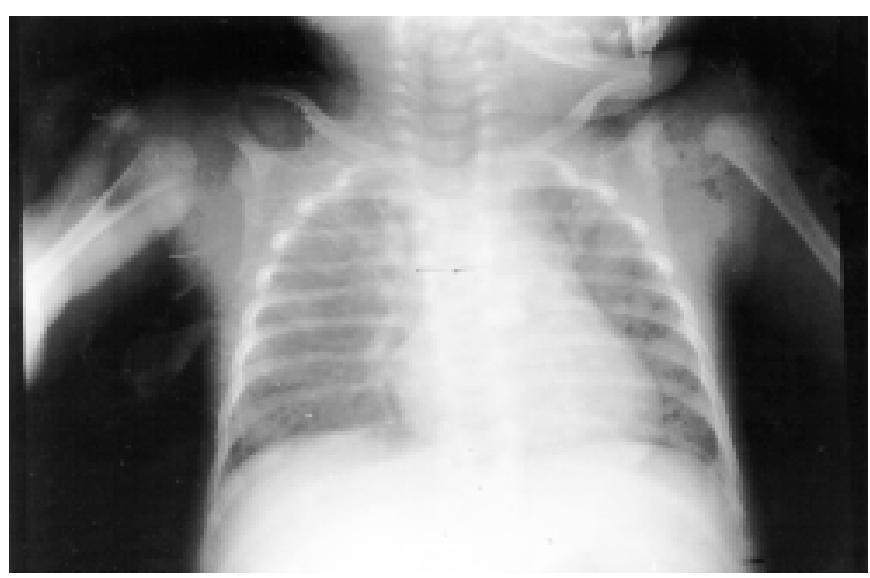

Fig. 1 - Hiperinsuflação pulmonar e infiltração intersticial difusa

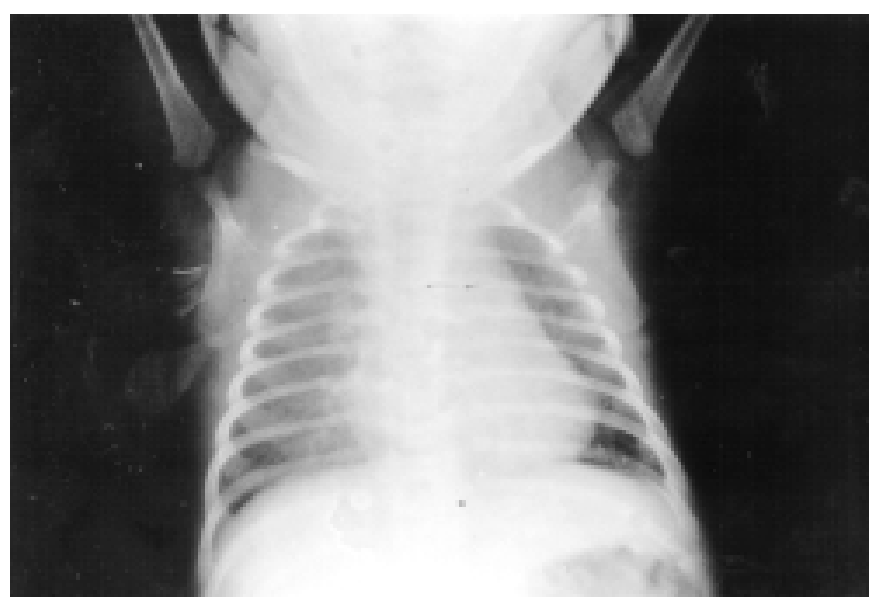

Fig. 2 - Infiltração intersticial difusa

Fisher $\mathrm{P}<0,0001)$ entre pneumonia e sorologia positiva (Tabela 3).

O diagnóstico radiológico é feito pela radiografia de tórax, onde é observada uma hiperinsuflação do parênquima pulmonar (Fig. 1) associada à infiltração intersticial difusa (Fig. 2). Neste trabalho o padrão descrito esteve presente em todas as crianças que tiveram pneumonia, no entanto o quadro radiológico poderá ser confundido com outras etiologias, principalmente virais, devendo ser lembrado no diagnóstico diferencial ${ }^{34}$.

O diagnóstico hematológico, como descrito na literatura, é de leucocitoce com eosinofilia; observamos, assim como Ejzemberg, em 199035, a presença de leucocitoce em sua maioria (Tabela 4). Não tem sido freqüente a eosinofilia, o índice neutrofílico nunca foi superior a 0,3 , o que o diferencia de outras infecções bacterianas.

\section{TRATAME NTO}

Como citado anteriormente, a profilaxia ocular real izada com nitrato de prata a $1 \%$ ao nascimento não afasta a infecção por $\mathrm{C}$. trachomatis e mesmo o uso de eritromicina a $0,5 \%$, já em sala de parto, poderá evitar a infecção ocular mas não previnirá a colonização em nasofaringe e a conseqüente infecção pulmonar. Os RN infectados por C. trachomatis devem ser tratados com eritromicina (etilsuccinato) na dose de $40 \mathrm{mg} / \mathrm{ki}$ lograma/dia divididos em quatro doses, durante 14 dias, incl usive para quem teve conjuntivite isolada, pois só o tratamento local com eritromicina falha em 50\% dos casos, recidivando. A eritromicina diminui significativamente o curso clínico da doença, a melhora ocorre ao redor do sétimo dia de tratamento e não são observadas recaídas ${ }^{30,36}$. Os RN deste estudo foram tratados com eritromicina (16/ 20) e os quatro que receberam trimetoprim-sulfisoxazol pelo fato de suspeita de pneumonia por Pneumocistis carinii.

\section{PRE VE NÇÃO}

O tratamento da mãe com a eritromicina antes do parto deverá prevenir a transmissão para o RN, e, já que não é toxica para o feto, deve ser administrada à mãe particularmente durante o terceiro trimestre de gestação e ao seu parceiro sexual. Os pais dos pacientes da presente casuística foram encaminhados para tratamento.

\section{CONCLUSÕES}

Salientamos a importância da pesquisa de infecção por C. trachomatis em RN com conjuntivite e/ ou pneumonia do tipo intersticial, mesmo na ausência de fatores de risco para doença sexualmente transmissível. A pesquisa direta em raspado de conjuntiva e o exame sorológico se mostraram importantes como métodos auxiliares do diagnóstico.

\section{SUMMARY}

\section{Chlamydia trachomatis infection in neona- tal period. Clinical and Laboratorial aspe- cts. Experience of ten years: 1987-1998.}

Chlamydia trachomatis infection is adquired by the newborn infant during the delivery, 25 to $50 \%$ of them may devel op conjunctivitis and 10 to $20 \%$ pneumonia.

BACKOGROUND. To verify the incidence of ocular infection by $C$. trachomatis in the newborn infants with conjunctivitis. To observe the association between ocular infection and intersticial pneumonia.-Study the epidemiological aspects and laboratorial methods of criterial diagnosis.

Casuistics and Methods. We studied the new- 
born infants admitted in the intensive neonatal care with diagnostic of conjunctivitis and/ or interstitial pneumonia during the period of ten years. The diagnostic methods were direct exam of etiologic agent in conjunctival material, $X$ ray chest and serologic test by imunofluorescence method for IgG and IgM antibodies.

RESULTS. We studied the clinical characteristics of 20 newborns infants with chlamydial trachomatis infection: 15 (75\%) were terms newborns and $5(25 \%)$ pre-terms. We observed the predominance of infection in females (60\%); pneumonia was observed in 15/ 20 (75\%) and 12 of them had both: conjunctivitis and pneumonia. we did not observe significant association among type of delivery, age of the mother, number of partner and infection. Leukorrhea was present em $50 \%$ of the mothers The serologic test was positive in $100 \%$ of the newborn with pneumonia and none with conjunctivitis alone, and the direct exam in conjuntival material was positive in newborns with conjunctivitis. The incidence of $\mathrm{C}$. trachomatis in the newborns admitted in this period with conjunctivitis were 17/ 100 (17\%).

Conclusion. Chlamydia trachomatis is an important pathogenical agent and the research of it is essential in newborn infants with conjunctivitis and/ or interstitial pneumonia even there were not risk factors for sexually transmitted diseases. The direct exam of conjunctival material and serologic test are very important to diagnosis. [Rev Ass Med Bras 1999; 45(4): 303-11]

KEY WORDS: Chlamydia trachomatis. Neonatal conjunctivitis. Interstitial pneumonia.

\section{REFERÊNCIAS BIBLIOGRÁFICAS}

1. Grayston J r, J .T., Wang S P - New Knowledge of chlamydiae and the diseases they cause. J Infect Dis 1975; 132: 87-105.

2. Campbell L A, KuoC C, Grayston J Y - Characterization of the new Chlamydia agent, TWAR, as a unique organism by restriction endonuclease analysis and DNA-DNA hybridization. J Clin Microbiol 1987; 25: 1911-5.

3. Wilfert, G M, Gutman L T - Chlamydia trachomatis infections in infants and children. Adv Pediatr 1986; 33: 49-75.

4. Rettig PJ - Perinatal I nfections with Chlamydia trachomatis. Clin Perinatol 1988; 15: 321-49.

5. Harrison H R, Alexander E R, Weinstein L - Cervical Chlamydia trachomatis and mycoplasmal infections in pregnancy: E pidemiology and out comes. J AM A 1983; 25: 1721-5.

6. Center for Disease Control. Chlamydia trachomatis infections MMWR 1985; 34: 53-74.

7. Fraser J J , Rettig P J , Kaplasn D W - Prevalence of cervical Chlamydia trachomatis and neisseria gonorrhoea in female adolescents. Pediatrics 1983; 71: 333-8.

8. Toomey K E, Raferti M P, Stamm W E - Unrecognizaed high preval ence of Chlamydia trachomatis cervical infection in na isolated Alaskan E skimo Population. J AMA 1987; 258: 53-9.
9. Brunham R C, Binns B, Mcdowell J et al. - Chlamydia trachomatis infection in women with ectopic pregnancy. Obstet Gynecol 1986; 67: 722-8.

10. Hammerschlag M R, Anderka M, Semine D Z et al. - Prospective study of maternal and infantile infection with Chlamydia trachomatis. Pediatrics 1979; 64: 142-8.

11. Rettig P J - Infection due to Chlamydia trachomatis from infancy to adol escence Pediatr I nfect Dis 1986; 5: 457-9.

12. Lascolea J J , Paroski J S, Buzindki J et al. - Chlamydia trachomatis in infants delivered by cesarean section. Clin Pediatr 1984; 23: 118-23.

13. Goldenberg R L, Andrews W W, Yuan A C et al. - Sexually transmitted diseases and adverse outcomes of pregnacy. Clin Perinatol 1997; 24: 23-41.

14. Alexander E R, Harrison H R - Role of Chlamydia trachomatis in perinatal infection. Ver Infect Dis 1983; 5: 713-8.

15. Frommel G T, Rothenberg R, Wang S - Chlamydial on infections of mothers and their infants. J Pediatr 1979; 95:28-33.

16. Heggie A D, Lumicao C G, Stuart L - Chlamydia trachomatis infection in mother and infants. AmJ DisChild 1981; 135: 507-15.

17. Bell T, Star W, Wang P - Chronic Chlamydia trachomatis infection in infants. J AMA 1992. 267: 400.

18. Harrison H R - Chlamydia trachomatis and chronic childhood lung disease. Pediatr Infect Dis 1982; 1: 395-401.

19. Pearson K, Ronnerstam R, Sanberg L - Neonatal Chlamydial eye infection: an epidemiological and clinical study. $\mathrm{Br}$ J Ophtalmol 1983; 67:700-4.

20. Givner L B, Rennels M B, Woodward C, Brenner J I-Chlamydial trachomatis infection in infant delivered by cesarean section. Pediatrics 19 81; 68:420-1.

21. Berman S M, Harrison H R, Boyce W T et al. - Low birth weight, prematurity and pos- partum endometritis. Associated with prenatal cervical Mycoplasma hominis and Chlamydia trachomatis infections. J AMA 1987; 1189-96.

22. Forster RK, Dawson CR, Sachachter J - Late follow up of patients with neonatal inclusion conjuntivitis. Am J Ophtalmol 1970; 69-467-70.

23. Laurenti R - Alguns aspectos particulares referentes aos resultados da I nvesti gação I nteramericana deM ortalidadena Infância na área do Projeto de São Paulo. In: Organização Mundial da Saúde- I nvestigação da mortalidadena infância do Brasil:Descobertas e atividades. Washington, OMS. 1977 (Publicação científica 343) p30.

24. BlytheM I, Katz B P, Batteiger B E - Recurrent genitourinary Chlamydial infections in sexually active femal eadolescents. J Pediatrics 1992; 14: 487.

25. Schachter, J , Grossman M , Seet R L - Prospective study of perinatal transmission of Chlamydia trachomatis. J AMA 1986; 225: 3374- 7.

26. Beem M O, Saxon E M- Respiratory tract colonization and a distinctive pneumonia syndrome in infants infectes with Chlamydia trachomatis. N Engl J Med 1977; 296:306-9.

27. Darougar $\mathrm{S}$ - The humoral immune response to Chlamydial infection in humans. Ver Infect Dis 1985; 7: 726-9.

28. Schachter J, Grossman M, Holt J - Prospective study of chlamydial infections in neonates. Lancet 1979; 2: 377-81.

29. Attenburrow A, Barker C M - Chlamydial pneumonia in the low-birthweight neonate. Ach Dis Child 1985; 60: 1169-73.

30. Tipple MA, Beem M O, Saxon E M - Clinical characteristics of the afebrile pneumonia associated with Chlamydia trachomatis infection in infants less than 6 month of age. Pediatrics 1979; 63:192-7.

31. Beem M O, Saxon E M-Chlamydia trachomatis infections of infants. In: Chlamydial Infections. Amsterdaam, Elsevier, Biomedical Press, 1982 p. 199. 
32. Schachter J, Grossman M, Azimi P H - Serology of Chlamydia trachomatis in infants. J infect Dis 1982; 146: 530-5.

33. Finn M P, Ohlin A, Schacter J - Enzime-linked immunosorbent assay for imunoglobulin $\mathrm{G}$ and $\mathrm{M}$ antibodies to Chlamydia trachomatis in human sera. J Clin Microbiol 1983; 17: 848-5.

34. Stagno S, Brasfield M, Brown M B et al. - Infant pneumonitis associated with cytomeglovirus, chlamydia, pneumocystis and ureapplasma: a prospective study. Pediatrics 1981. 68: 322-9.

35. Ejzenberg B - Contribuição ao estudo das infecções causadas por Chlamydia trachomatis em crianças entre 1 e 6 meses internadas com pneumonia. São Paulo, 1990 (Tese de Doutorado-facul dade de Medicina da USP) p. 213.

36. Beem M O, Saxon E, Tipple M - Treatment of Chlamydial pneumonia of infancy. Pediatrics 1979; 63: $198-203$. 